\title{
Characterization of abnormal grain coarsening in Alloy 718
}

\author{
Richard Watson ${ }^{1, a}$, Michael Preuss ${ }^{1}$, João Quinta da Fonseca ${ }^{1}$, Thomas Witulski², Gregor Terlinde ${ }^{2}$, and Markus Büscher ${ }^{2}$ \\ ${ }^{1}$ University of Manchester, School of Materials, Grosvenor Street, Manchester M1 7HS, UK \\ ${ }^{2}$ Otto Fuchs KG, Abt. 05/2, Derschlager str. 26, 58540 Meinerzhagen, Germany
}

\begin{abstract}
Even though the phenomenon of abnormal grain coarsening (AGC) or "exploded grains" has been known to occur in Alloy 718 industrial forgings there is still no satisfactory explanation for it. For this reason, detailed microstructure analysis has been carried out in normal and abnormal regions. Electron Backscatter Diffraction (EBSD) was employed to determine grain size, boundary distribution and measure stored energy, while backscattered imagining in a FEGSEM was used to measure $\delta$ precipitate size and morphology. It was found that abnormal regions show almost 3 times as many twin boundaries compared to a normal region. In addition, the $\delta$ phase morphologies differ very significantly when comparing these two different regions. Normal regions display $\delta$ phase with a plate like nature, whereas in abnormal regions, $\delta$ particles appear to be more spherical. Furthermore, there are clear indications of differences in $\delta$ volume fractions between the two regions. Whilst in normal regions the $\delta$ phase is found predominantly at grain boundaries, in abnormal regions the $\delta$ is also found within grains. Both backscatter images and EBSD scans indicate that there are higher levels of stored energy within the normal regions, compared to the abnormal regions. These observations suggest that AGC occurs in regions where dynamic recrystallization does not happen and where recrystallization during solution heat treatment is affected by the local particle distribution.
\end{abstract}

\section{Introduction}

Due to its good weldability, corrosion resistance, high strength and good fatigue life, Alloy 718 has found uses in a variety of applications in Aerospace and oil and gas. Whilst there have been a variety of papers outlining the ideal microstructures for forged Alloy 718 papers with extensive information on forging parameter optimization [1-4], what has not been so well documented is the occurrence of undesired microstructures and in particular the conditions under which these undesired microstructures form.

A few papers do exist on the effect of abnormal grain growth (AGG) in Superalloys. Bozzolo et al. showed that in low strain torsion tests Alloy 718 produced abnormally large grains that grew unhindered despite the presence of $\delta$ phase particles [5]. The large grains seen in this study had roughly twice the amount of twin boundaries than normalsized grains, appearing scattered throughout a low strained part of the microstructure. This phenomenon is not just limited to Alloy 718 but has also been shown to occur in Udimet 720 derivatives [6].

Flageolet et al. studied the effect of abnormally coarsened grains on the LCF (low cycle fatigue) and tensile properties of Alloy 718 [7]. The work showed little effect of the abnormal microstructure on the tensile properties at $350{ }^{\circ} \mathrm{C}$ but did adversely affect the LCF of the material. This study also showed through the use of EBSD that the

a Corresponding author: richard.watson-2@postgrad. manchester.ac.uk abnormal regions contained a higher proportion of twins than the normal regions.

Quantification of abnormally coarse microstructures in Alloy 718 is difficult and can be subjective. Usually, the only way to obtain good quantitative information of twin volume fraction is through EBSD. Papers on grain boundary engineering do offer some characterisation techniques, but these usually only focus on the proportion of twin boundaries to other high angled grain boundaries and the amount of triple points that contain two or more twin boundaries to reduce crack propagation, often failing to characterise grain morphology $[8,9]$.

Some efforts to characterise and predict twinned microstructure have been made in Nickel. Mahajan et al. suggested that there are four types of annealing twin that can occur in f.c.c metals and gives a theoretical explanation on twin growth [10]. Pande et al. showed that increased grain boundary velocity gives rise to more opportunities for twins to occur [11]. Wang et al. showed that the number of twins per grain decreases with increasing deformation in a Nickel base alloy [12]. These papers are however more focused with predicting and quantifying the amount of annealing twin boundaries present than the microstructures that produce them.

The present paper aims to characterise a more extreme form of undesired microstructure observed in an Alloy 718 trail forging. The highly twinned abnormal microstructures manifest during the solution heat treatment stage of Alloy 718 component production in areas that undergo only small amounts of deformation. The main issue with 


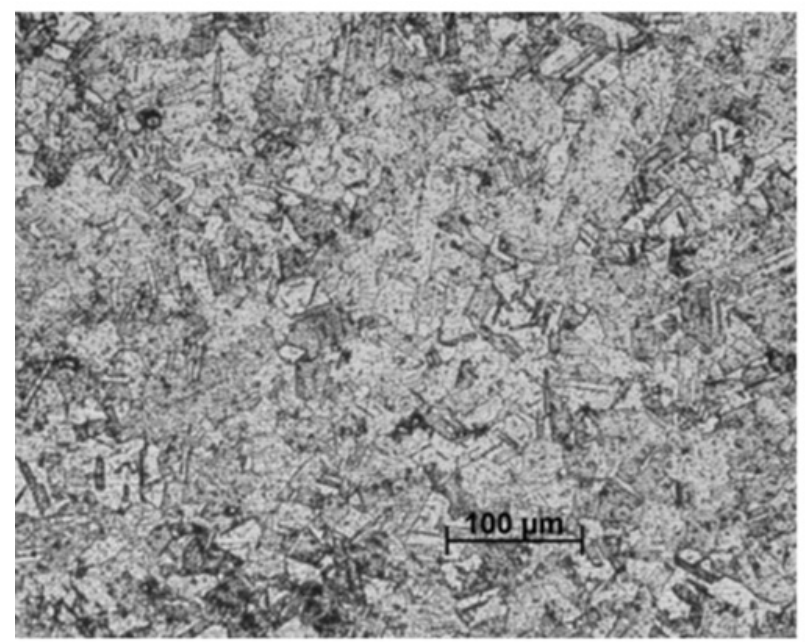

(a)

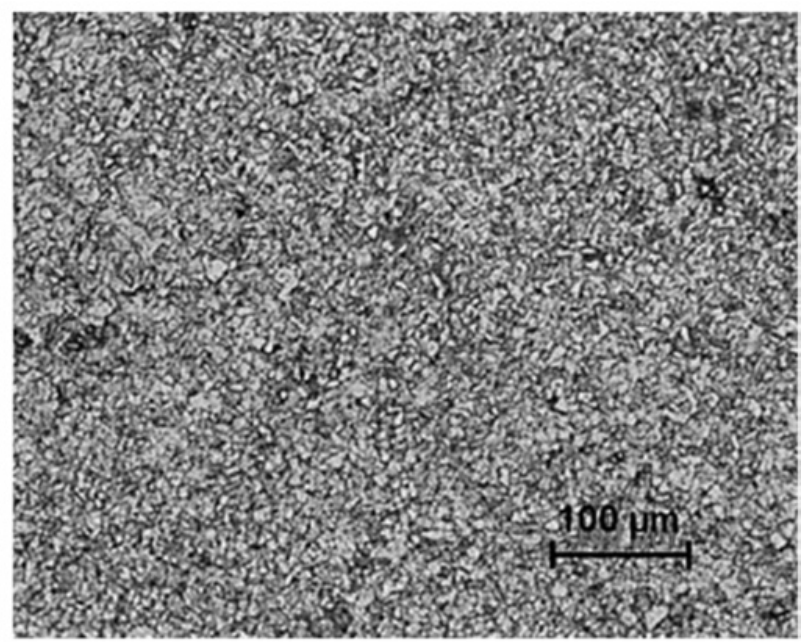

(b)

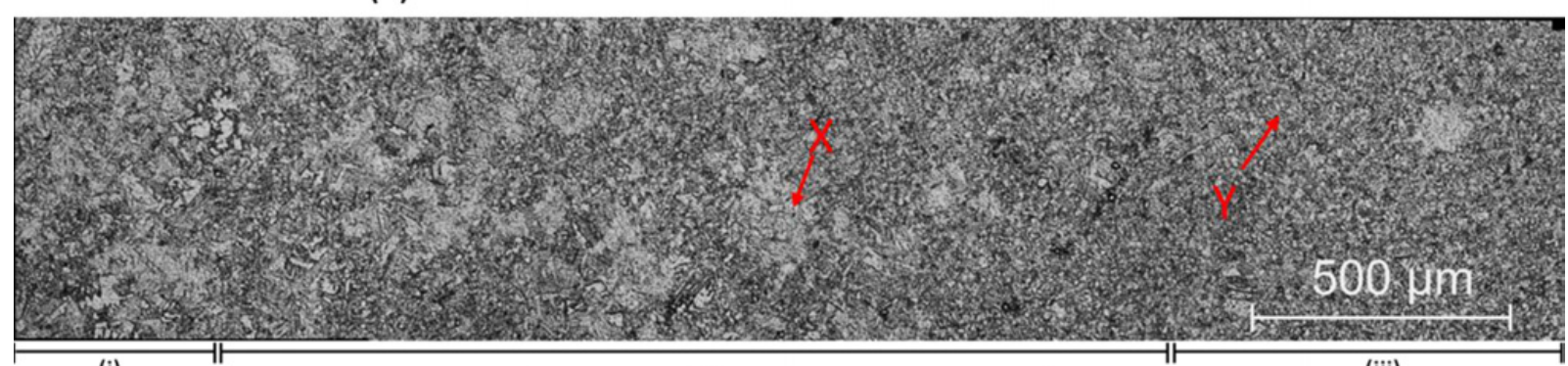

(ii)

(iii)

(c)

Figure 1. Optical images of Alloy 718 showing an abnormal region (a) normal region (b) and an evolution from abnormal region on the left (i) to transition region (ii) to normal region on the right (iii) (c). In (c) $\mathrm{x}$ is representative of an AGC zone and $\mathrm{y}$ indicates a normal region.

the observed microstructure is that it is not predicted by current microstructure models. For the purpose of this investigation the phenomenon will be referred to as Abnormal Grain Coarsening (AGC) rather than Abnormal grain growth (AGG) as the exact mechanism behind their growth is uncertain.

\section{Experimental}

The investigation was carried out on a section of a trial Alloy 718 disc forging provided by Otto Fuchs. The part had undergone three forging steps each done below the $\delta$ solvus, followed by a solution heat treatment below the $\delta$ solvus then a polymer quench and finally a two-step aging process

For optical microscopy, samples were first ground, diamond polished and further polished with colloidal silica suspension (OPS) for $4 \mathrm{hrs}$ before using the Kalling 2 Microetchant. In preparation for BSE imaging and EBSD in the scanning electron microscope (SEM), the samples were not etched. The size of the area investigated using EBSD for both regions were determined from the optical and back scatter images. The step size for the EBSD was $1 \mu \mathrm{m}$ in AGC regions and $0.5 \mu \mathrm{m}$ elsewhere. The scan was conducted at a working distance of $10 \mathrm{~mm}$ and accelerating voltage of $20 \mathrm{kV}$ using the HKL system on a FEI Sirion Field Emission Gun Scanning Electron Microscope.

\section{Results}

\subsection{Grain morphology analysis}

All samples that develop AGC come from regions of low strain. The optical images show the differences between a region abnormal Fig. 1a and normal grain size regions Fig. 1b. It can be seen that it is comparatively difficult to distinguish individual grains in the abnormal region resulting in significant uncertainty when undertaking grain size analysis from optical micrographs. Figure 1a also indicates that there is a significant amount of twin boundaries in the abnormally coarse grain regions. Fig. 1c shows the transition between abnormal to a normal region. The transition occurs over a relatively small distance, approximately $4-5 \mathrm{~mm}$, with some isolated coarse grains forming in the normal zone, the fraction of coarse grains rapidly increases from the right to the left side.

Figure 2 shows BSE images more detailed images of the two regions and the transition zone. These images show more clearly the high twin volume fraction and also reveal intragranular $\delta$ phase particles showing up as white dots within the larger abnormally sized grains. It is interesting 


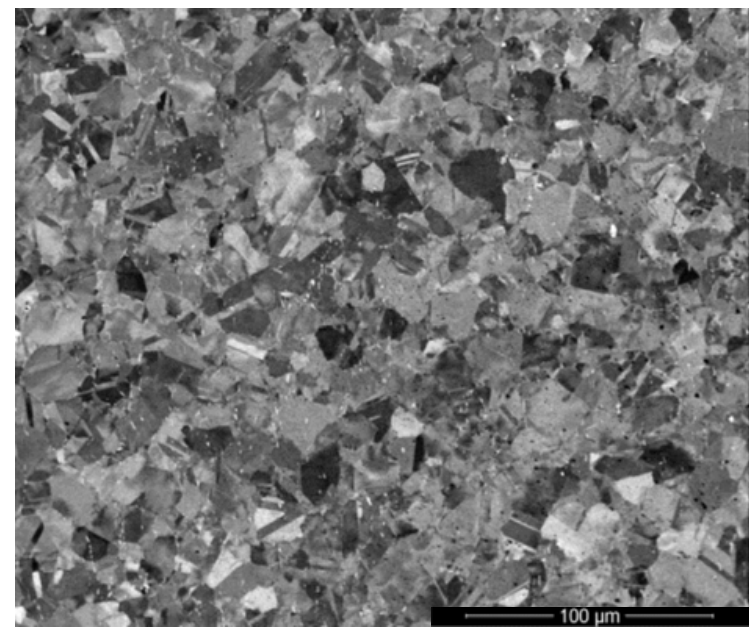

(a)

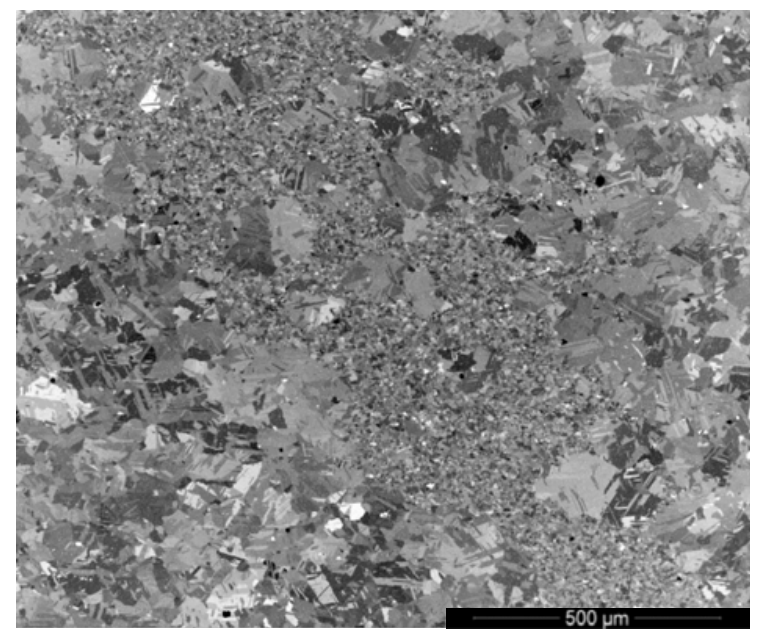

(c)

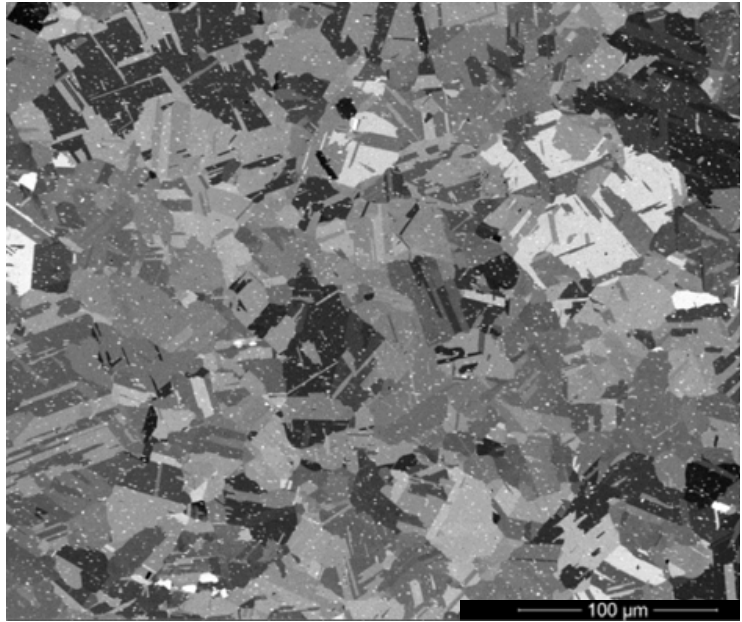

(b)

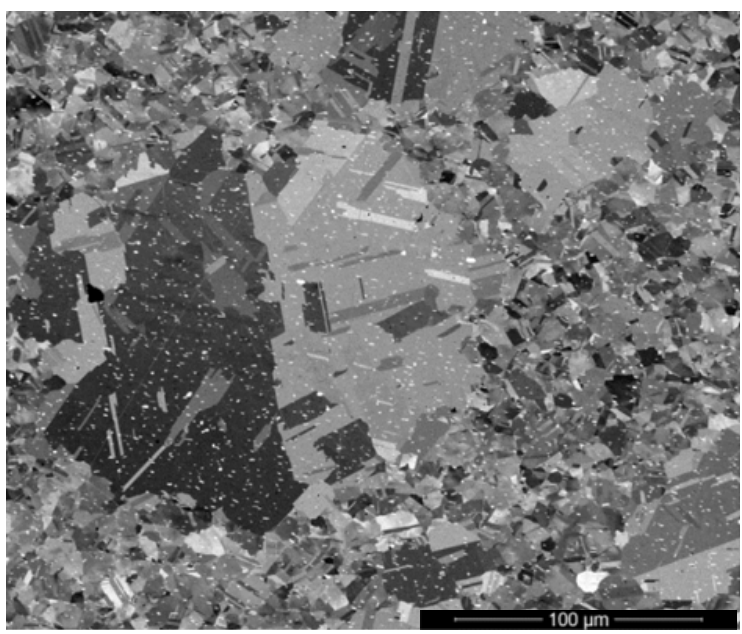

(d)

Figure 2. Backscatter electron images from normal and abnormal grain size regions within an Alloy 718 trial forging. A normal microstructure for Alloy 718 is shown in (a), (b) shows a general overview of an abnormal region where the $\delta$ phase can be observed to be intergranular, (c) transition zone between normal and abnormal grain size region, and (d) particularly coarse grain next to normal sized grains with intragranular $\delta$ phase clearly visible as white dots.

to note that in the normal-size grain region individual grains contain a range of different grain shades whereas abnormal size grains tend to display only a single shade of grey. This contrast is an indication of lattice distortion and therefore stored energy.

\subsection{Grain orientation analysis}

Figure 3 displays inverse pole figure orientation maps recorded by EBSD of an abnormal grain size region interspersed with some normal-size grains for Alloy 718. In both cases a good array of grain orientations can be observed suggesting no strong crystallographic microtexture in these regions. When these and other EBSD scans were analysed it was found that the percentage twin boundaries in the abnormal grain size zones was $66 \%$ opposed to $24 \%$ in the normal-size grain size regions.

Figure 4 shows binary images created from backscatter electron images highlighting the $\delta$ phase in an abnormal grain size region (a) and normal grain size region (b) recorded in Alloy 718. There is a clear difference between the two with the $\delta$ in the abnormal grain size region being larger and more spherical in shape than the typical $\delta$ platelet formation observed in the normal grain size region. Another difference is that in the $\delta$ phase in abnormal regions sits intragranular whilst $\delta$ in normal regions is found at grain boundaries. It is also notable that the intragranular $\delta$ precipitates in the abnormal grain size region are arranged as if they had precipitated on prior grain boundaries.

Figure 5 plots maps of the average misorientation for individual grains in (a) the normal and (b) abnormal grain size regions in Alloy 718 to highlight the differences in stored energy between the two. This method takes the average of all the pixel misorientations across the grains and applies it to the whole grain. It can be clearly seen in Fig. 5a that in the normal grain size region the large grains display a very noticeable level of misorientation within the grains while the finest grains do not. In contrast, Fig. 5b 


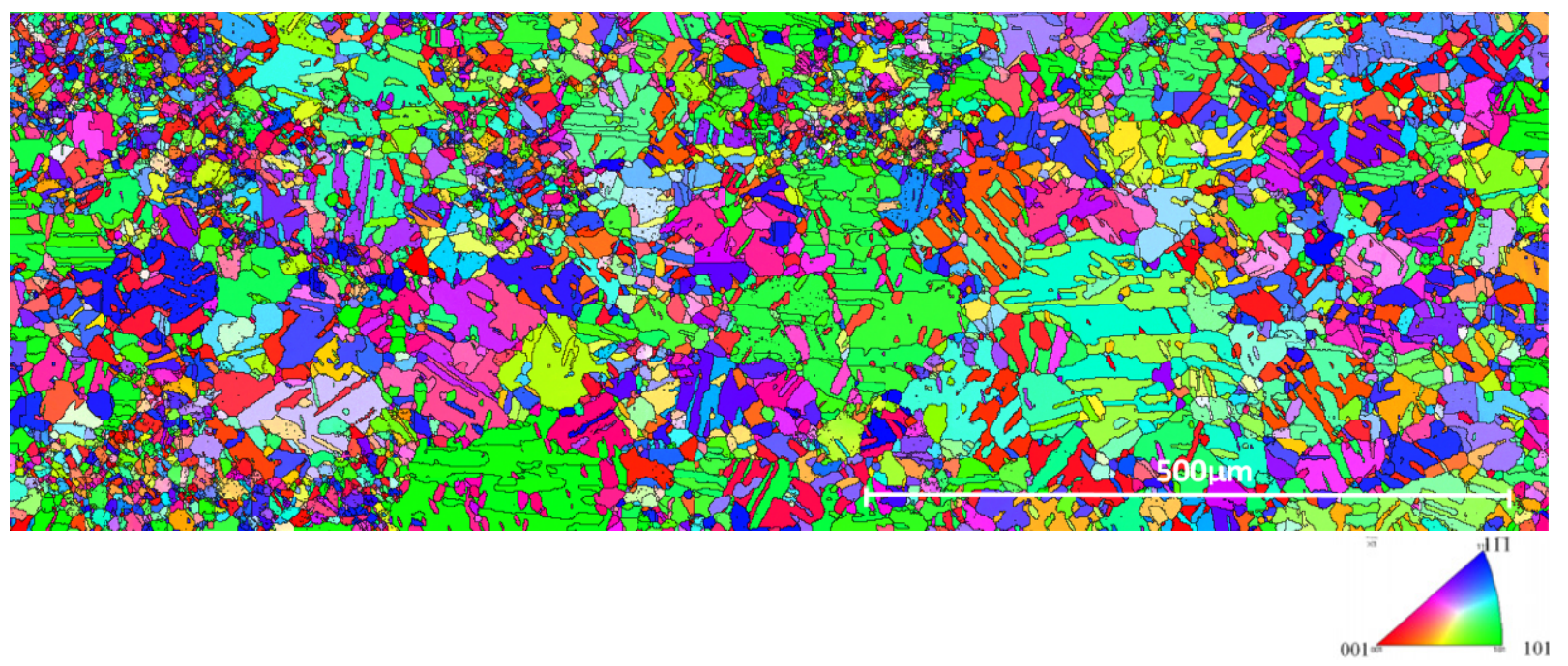

Figure 3. IPF colour maps showing an abnormally coarse grain size region in an Alloy 718 trial forging interspersed with normal-size grains.

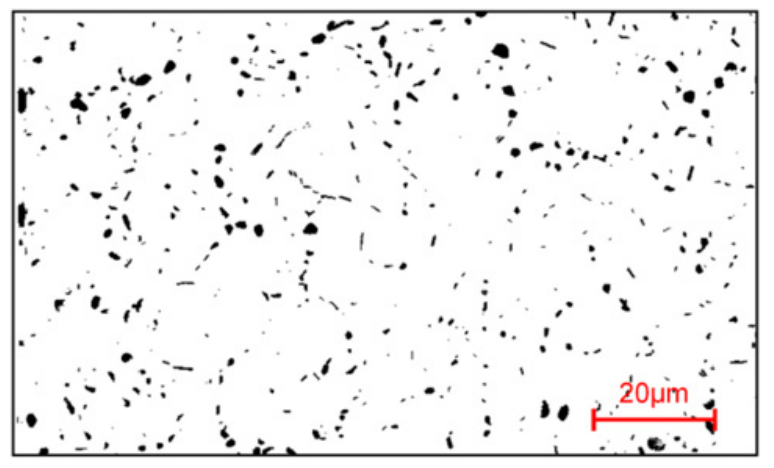

(a)

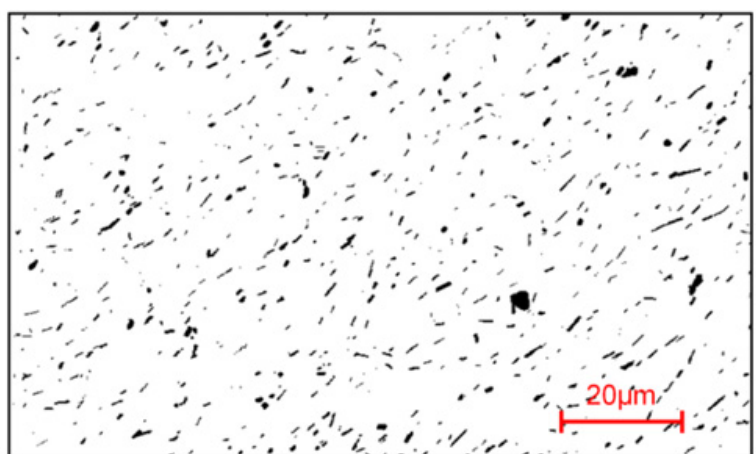

(b)

Figure 4. Binary images of the $\delta$ phase created from BSE images from (a) abnormal grain size region and (b) normal grain size region.

demonstrates misorientation levels close to zero for most grains in the abnormal grain size region.

\section{Discussion}

Both BSE and EBSD analysis show that the abnormal grain size region displays a significantly higher twin volume fraction and lower levels of grain misorientations compared to the normal grain size regions. Due to the straight nature of the boundaries and conditions under which they were formed, the twins in the backscatter images of the abnormal grain size regions are thought to be annealing twins rather than deformation twins. The lower level of grain misorientation suggests that in the abnormal grain size region an exceptional low level of stored energy is achieved.

An interesting observation is that the $\delta$ phase in Alloy 718 does not pin the grain boundaries in the coarse grain region as they are found to be intragranular. What is also worth noting is that when there is a twin boundary there is usually one or more $\delta$ phase particles in contact with this boundary. As the $\delta$ phase nucleates and growths along the $\{111\}$ planes, which is also the twin boundary plane, there could be a possible link between particles and twin growth.
The average grain misorientation results in Fig. 5 show a significant difference in stored energy between the normal and abnormal grain size regions despite both regions being heat treated after forging. Samples of the alloy came from regions of low strain during forging. Simulations and previous studies [5,6] suggest that the critical strain for abnormal grain coarsening during solution heat treatment is less than 0.2. However initial tests on this material have produced negative results. This suggests that the condition under which these grains from is a lot more complex.

At this stage one can only speculate how this situation could arise. There are three main observations, differing $\delta$ distribution, different twin volume fraction and differing mean grain orientation. As the $\delta$ particles are intragranular in the abnormal region this suggests that the driving force for grain boundary movement was higher in the AGC regions. The disappearance of the stored energy indicates that this is potentially where the higher driving force originates from and the high volume of twin boundaries implies that the mechanism is driven by the growth and movement of twin boundaries.

This work suggests that the true mechanistic explanation of the abnormal grain coarsening behaviour in Nickel 


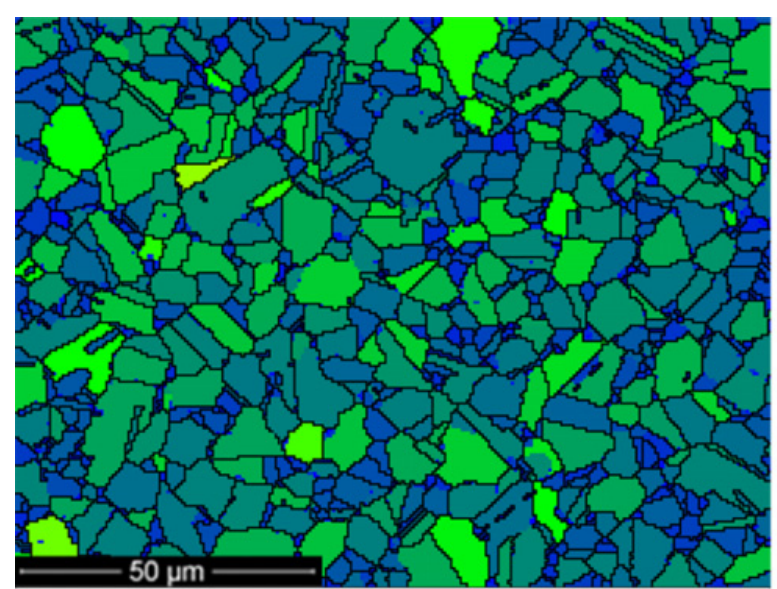

(a)

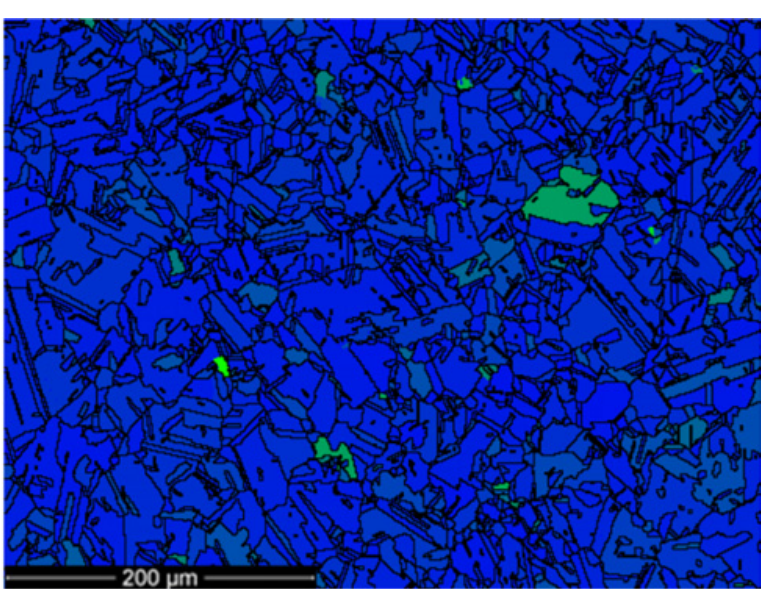

(b)

$1^{\circ}$

$2^{\circ}$

$3^{\circ}$

$4^{\circ}$

$5^{\circ}$

Figure 5. Average misorientation of grains for (a) normal grain size region and (b) abnormal grain size region in Alloy 718 .

Superalloys will require a much improved understanding of the formation of annealing twins. Work by Mahajan et al. [10], that was based on the work of Glieter [13], Dash and Brown [14] and Meyers and Murr [15] has suggested theoretical models of how twining may occur in f.c.c materials. This work suggests that nucleation of Shockley partials on steps at grain boundaries that coincide with $\{111\}$ planes lead to formation of annealing twins. This model assumes that the grain boundary is moving and growth accidents can occur on these moving boundaries. If the Shockley partials are of the same type they repel each other and glide away from the boundary into the grain producing a one layer thick twin. This then changes the habit plane of the grain boundary leading to further partial loops forming and then propagating into the grain. If the grain boundary velocity is high then there is a greater probability that growth accidents can occur leading to increased twinning. The likelihood is that conditions exist in these forgings that allow for rapid grain growth and increased occurrence of growth accidents leading to twinning.

\section{Conclusions}

1) Abnormally coarse and highly twinned microstructures have been observed in an Alloy 718 trial forging. The twins appear to be annealing twins rather and deformation twins due to the straight nature of the boundaries, existence of incoherent twin boundary fronts and the fact that they have been observed after a high temperature heat treatment.

2) EBSD scans give more detail on the orientation of the grains showing no dominating texture in the coarse grain region.

3) A difference in stored energy has also been observed through the use of grain surface misorientation plots, showing that there is less stored energy within the AGC regions compared to the standard condition.

4) It was found that $\delta$ precipitates fail to pin grain boundaries in the AGC region.

\section{References}

[1] Mataya M, Matlock D. Superalloy 718 - Metall Appl, 155-178 (1989)

[2] Brooks J.W, Bridges P. Superalloys 1988 33-42 (1988)

[3] Dedvallees Y, Bouzidi M, Bois F. Superalloys 718, 625, 706 Var Deriv, 281-291 (1994)

[4] Wilkinson NA. Superalloy 718 - Metall Appl, 119-133 (1989)

[5] Bozzolo N, Souaï N, Logé R, Agnoli A, Bernacki M. 5th Int Conf Recryst Grain Growth (2013)

[6] Bozzolo N, Souaï N, Logé RE. Acta Mater, 60, 5056-5066 (2012)

[7] Flageolet B, Yousfi O, Dahan Y, Villechaise P, Cormier J. Superalloy 718 Deriv, 595-606, (2010)

[8] Krupp U, Kane WM, Liu X, Dueber O, Laird C, McMahon CJ. Mater Sci Eng A, 349, 213-217 (2003)

[9] Detor A, Deal A, Hanlon T. Superalloys 2012, 873-880 (2012)

[10] Mahajan S, Pande C, Imam M, Rath B. Acta Mater, 45, 2633-2638 (1997)

[11] Pande C, Imam M, Rath B. Metall Trans A, 21, 2891-2896 (1990)

[12] Wang W, Brisset F, Helbert a. L, Solas D, Drouelle I, Mathon MH, Baudin T. Mater Sci Eng A, 589, 112-118 (2014)

[13] Gleiter H. Acta Metall, 17, 1421-1428 (1969)

[14] Dash S, Brown N. Acta Metall, 11, 1067-1075 (1963)

[15] Meyers M, Murr L. Acta Metall, 26, 951-962 (1978) 